\title{
An Information Theoretical Analysis of Broadcast Networks and Channel Routing for FRET-based Nanoscale Communications
}

\author{
Murat Kuscu Derya Malak Ozgur B. Akan \\ Next-generation and Wireless Communications Laboratory (NWCL) \\ Department of Electrical and Electronics Engineering \\ Koc University, Istanbul, Turkey \\ Email: \{mkuscu,dmalak, akan\}@ku.edu.tr
}

\begin{abstract}
Nanoscale communication based on Förster Resonance Energy Transfer (FRET) enables nanomachines to communicate with each other using the excited state of the fluorescent molecules as the information conveyer. In this study, FRET-based nanoscale communication is further extended to realize FRETbased nanoscale broadcast communication with one transmitter and many receiver nanomachines, and the performance of the broadcast channel is analyzed information theoretically. Furthermore, an electrically controllable routing mechanism is proposed exploiting the Quantum Confined Stark Effect (QCSE) observed in quantum dots. It is shown that by appropriately selecting the employed molecules on the communicating nanomachines, it is possible to control the route of the information flow by externally applying electric field in FRET-based nanonetworks.
\end{abstract}

\section{INTRODUCTION}

A number of nanomachines communicating with each other is envisioned as a nanonetwork. Nanonetworks allow nanomachines to cooperatively share information to achieve a specific task ranging from nuclear defense to drug delivery. [1]. The shared information among nanomachines can be either an output of a sensing process or a control signal coming from an optical or electrical source and intending to control the operation of nanomachines by giving specific orders. In both cases, designing and modeling information sharing mechanisms among nanomachines and investigating the methods for controlling the route of the information flow is crucial.

In recent years, several potential approaches have been proposed in order to achieve communication in nanoscale such as electromagnetic, nanomechanical or molecular [1][3]. In addition to existing nanoscale communication techniques, recently, a novel and radically different method has been proposed exploiting a well known phenomenon, Förster Resonance Energy Transfer (FRET) [4].

FRET is a non-radiative energy transfer process between fluorescent molecules, e.g., organic dyes, fluorescent proteins,

This work was supported in part by the Turkish Scientific and Technical Research Council under grant \#109E257, by the Turkish National Academy of Sciences Distinguished Young Scientist Award Program (TUBA-GEBIP), and by IBM through IBM Faculty Award. and semiconductor nanoparticles those have a significant spectral overlap and located in a close proximity such as 0 to $10 \mathrm{~nm}$ [5]. The phenomenon is widely used in studies of biotechnological research including fluorescence microscopy, molecular biology and optical imaging, since it yields a significant amount of structural information about the molecules [6].

The FRET-based nanoscale communication system model proposed in [4] uses pulsed laser with picoseconds duration pulses as the Information Source (IS) and implements ONOFF keying (OOK) modulation with two bits available. When the information source intends to transfer bit 1 , a single laser pulse is sent to the Transmitter Nanomachine (TN) at the beginning of a specific-duration time slot. The fluorescent molecule on TN acting as a donor is excited by this pulse and after some time it relaxes through either fluorescence or FRET by transferring its excited energy to a nearby acceptor molecule on a Receiver Nanomachine (RN) and making the acceptor excited. RN is assumed to have the ability of detecting the state of the acceptor molecule instantly. If FRET occurs following the excitation of the donor, $\mathrm{RN}$ detects the acceptor's excited state and decides bit 1 . In the bit 0 case, IS does not send any pulse to the TN and keeps silent in a time slot duration. Therefore, the acceptor on RN stays in the ground state and RN detects bit 0 . The proposed channel is analyzed information theoretically in the same study, and the dependence of the channel capacity on some environmental and intrinsic parameters, e.g., internodal distance, optical spectra of the employed molecules, and refractive index of the medium, is investigated and it is shown that by appropriately selecting the parameters, it is possible to realize point-to-point communication with high communication capacities in $0-10$ nm range.

In this study, using the point-to-point FRET-based communication channel proposed in our previous work [4], FRETbased broadcast channel is modeled and analyzed information theoretically with a new waveform representing bit 0 and bit 1 and providing reliable communication in several network scenarios. It is shown that, with the proposed waveform, reli- 


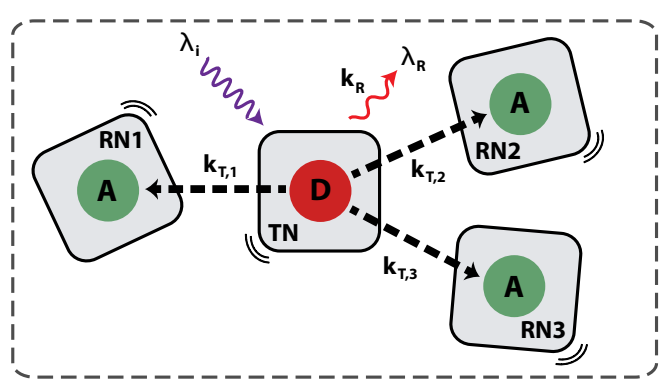

Fig. 1. Broadcast network with one TN communicating with three RNs.

able transmission of information from a single $\mathrm{TN}$ to several RNs can be realized. Furthermore, exploiting the Quantum Confined Stark Effect (QCSE) observed in QDs, it is possible to route the information flow by electrically controlling it in a nanonetwork based on FRET.

The remainder of this paper is organized as follows. In Section II, FRET-based broadcast communication with one TN and many RNs is investigated. In Section III, an electrically controllable routing method in FRET-based networks is proposed. An example network scenario comprising broadcasting, relaying and routing methods is analyzed information theoretically in Section IV. Finally, the concluding remarks are given in Section V.

\section{FRET-BASED BROADCAST COMMUNICATION}

In this section, FRET-based broadcast communication is investigated with one TN surrounded by many RNs in an arrangement as seen in Fig. 1, and the performance of single TN-RN pair in a broadcast nanonetwork is analyzed in terms of channel capacity. Considering one cycle of excitation and relaxation of the donor on the $\mathrm{TN}$, it is not possible for the donor to deliver its excited energy to all of the acceptors on RNs; actually, it can only excite one of the acceptors. Therefore, the transmission of bit 1 to all of the RNs is very problematic, i.e., the reliability of the broadcast channel is very low, with single pulse excitation, and more reliable waveform representing bit 1 and bit 0 is required.

\section{A. Pulse Train Representation of Information Signals}

A new waveform is proposed to improve the channel capacity and to make it possible to implement broadcasting in FRET-based communication network. The proposed scheme uses a pulse train comprising $N$ pulses instead of a single pulse to represent bit 1 . Bit 0 is represented by no pulse in a time slot as in the single pulsed scheme.

In the pulse train, the time period of the pulses are equal to the slot duration $T_{H}$ of the single pulsed scheme. Since, an excited fluorescent molecule cannot be re-excited until it relaxes, $T_{H}$ must be reasonably greater than $\tau_{L}+\tau_{D A}+\tau_{F R E T}+\tau_{A}$, in order to avoid intersymbol interference (ISI). Here, $\tau_{L}$ is the pulse duration, $\tau_{D A}$ is the average excited state lifetime of the donor molecule in the case of FRET, $\tau_{F R E T}$ is the average duration of the FRET process, and $\tau_{A}$ is the average excited state lifetime of the acceptor. Since $\tau_{D A}+\tau_{F R E T}$ is

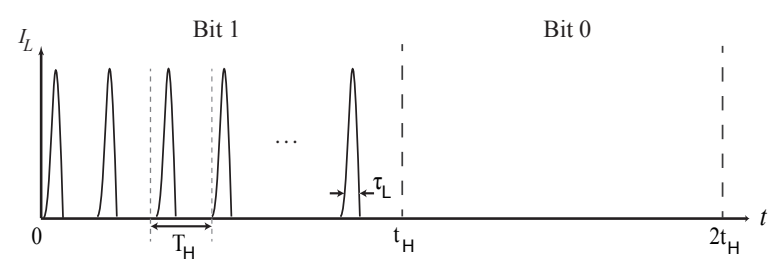

Fig. 2. Waveform representing bit 1 and bit 0 .

approximately equal to $\tau_{D}$, and $\tau_{L}$ is in picoseconds-range and negligible compared to nanoseconds-range lifetimes of donor and acceptor, the minimum value of time slot $T_{H, \min }$ can be given as $\tau_{D}+\tau_{A}$. Note that, the slot duration of the new excitation waveform $t_{H}$ must be greater than $N \cdot T_{H}$.

Since most of the fluorescent molecules have high absorption coefficient and actually vast amount of photons released from IS during a picoseconds-length pulse, the donor on the $\mathrm{TN}$ can be assumed to be excited by one of these photons during each laser pulse. Therefore, when bit 1 is sent from IS to TN, TN achieves to transmit bit 1 to the RN successfully with probability $p_{1}$. Assuming that RN can detect the excited state of the acceptor instantaneously and without error, $p_{1}$ is equal to the probability of FRET in a $t_{H}$-duration time slot and can be given as

$$
p_{1}=1-\left(1-P_{F R E T}\right)^{N}
$$

where $P_{F R E T}$ is the probability of FRET for a single pulse excitation and equal to the efficiency of FRET. In terms of internodal distance $R, P_{F R E T}$ is given as

$$
p_{F R E T}(R)=E_{F R E T}(R)=\frac{R_{0}^{6}}{R^{6}+R_{0}^{6}}
$$

where $R_{0}$ is the Förster distance which states the internodal distance when the efficiency is 0.5 . $R_{0}$ depends on some environmental and intrinsic parameters such as the spectral overlap of emission and absorption spectra of donor and acceptor molecules, respectively; refractive index of the medium and relative orientation of the dipole moments. Note that, it is sufficient for RN to detect one of the excited states of acceptor during $t_{H}$; therefore, once $\mathrm{RN}$ detects an excited state, it ignores the successive excitations and wait until the next time slot. The probability of successful transmission of bit 0 , i.e., $p_{0}$, is equal to 1 as in the case of single pulse excitation.

From the derived transmission probabilities, it can be concluded that, even a small $N$ is enough to realize a noiseless communication between two nanomachines which have an internodal distance around $R_{0}$. Note that, for the most of the fluorescent molecules, $R_{0}$ ranges between 4 and $7 \mathrm{~nm}$.

\section{B. FRET-based Nanoscale Broadcast Communications}

For an excited donor molecule and nearby ground-state acceptor molecule, the probability of FRET in terms of process rates can be expressed as

$$
P_{F R E T}=\frac{k_{T}}{k_{R}+k_{T}}
$$




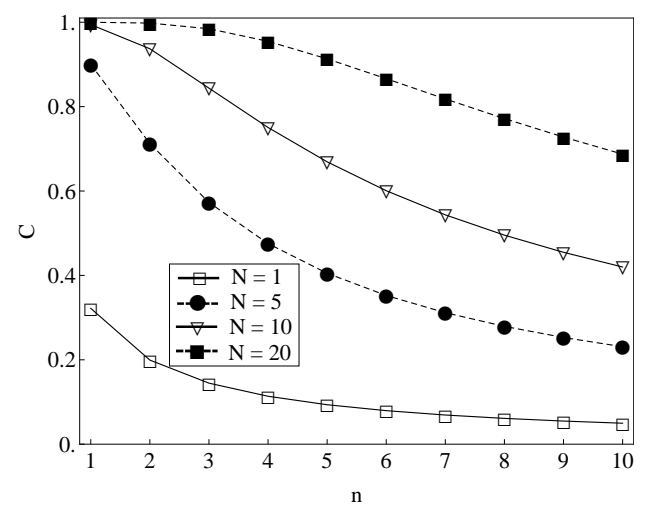

Fig. 3. Channel capacity $C$ with varying $n$ number of RNs for different $N$.

where $k_{R}$ is the fluorescence rate of the donor which is equal to $1 / \tau_{D}$, and $k_{T}$ is the FRET rate. In the case of several acceptors surrounding a single donor in a close proximity, excited donor either fluorescences or gets into resonance with one of the acceptors. Therefore, the probability of FRET between the excited donor and a specific acceptor can be given as

$$
P_{F R E T}=\frac{k_{T, i}}{k_{R}+\left(k_{T, 1}+k_{T, 2}+\ldots+k_{T, n}\right)}, 1 \leq i \leq n
$$

where $n$ is the number of acceptors, $k_{T, i}$ is the FRET rate between the donor and the $i^{t h}$ acceptor. $k_{T}$ strongly depends on $R$ and $R_{0}$, and can be expressed as

$$
k_{T}=\frac{1}{\tau_{D}}\left(\frac{R_{0}}{R}\right)^{6}
$$

For the same type of acceptors, i.e., with the same $R_{0}$ values, excited energy is transferred from the donor to the nearest acceptor with the highest probability. If the acceptors are located with the same distance to the donor, the probability of energy transfer from the donor to one of the acceptors becomes

$$
P_{F R E T}=\frac{k_{T}}{k_{R}+n k_{T}}
$$

From the communication aspect, a simple broadcast network can be constructed with a donor molecule bound to a TN and the same type of acceptor molecules bound to several RNs which are assumed to have equal distances to the TN as demonstrated in Fig. 1. Since the acceptor molecules are of the same type, their emission and absorption spectra have negligible overlap. Therefore, inter-acceptor FRET between an excited acceptor and a ground-state acceptor can be neglected. The donor on the TN is again assumed to be excited for each laser pulse coming from the IS. Applying pulse train waveform, the period of the pulses $T_{H}$ must be greater than $\tau_{D}+\tau_{A}$. Note that, if different type of acceptors are bound on RNs, then $T_{H}$ must be greater than $\tau_{D}+\tau_{A, \text { max }}$, where $\tau_{A, \max }$ the longest acceptor lifetime among the network. Using (1) and (6), the transmission probability of bit 1 to one out of $n$ number of RNs in a $t_{H}$-duration slot is given as

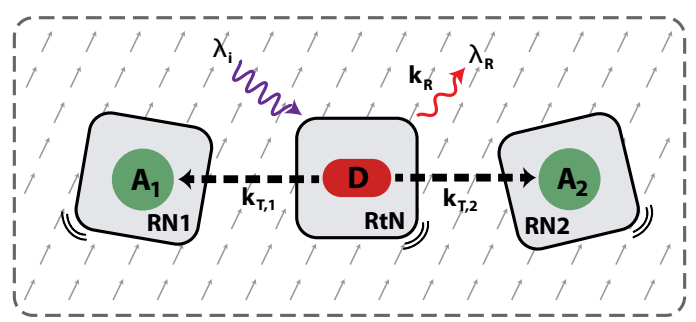

Fig. 4. Electrically controllable routing of the communication between one RtN and two RNs.

$$
p_{1}=1-\left(1-\frac{k_{T}}{k_{R}+n k_{T}}\right)^{N}
$$

The successful transmission probability of bit 0 , i.e., $p_{0}$, from the $\mathrm{TN}$ to one of the acceptors is 1 . Hence, from the information theoretical aspect, the broadcast channel is a Zchannel. The capacity of the channel is calculated as the maximum mutual information between the $\mathrm{TN}$ and each of the RNs and its dependence on the number of RNs in the network is demonstrated in Fig. 3 for different number of repetitions in the modulation assuming the distance between the $\mathrm{TN}$ and each RN is equal to Förster distance, i.e., $k_{T}=k_{R}$. As is seen, for single pulsed case, the capacity decreases under 0.2 bits for two and more RNs, since each time bit 1 is transmitted, only one of the RNs can receive the data in the specified time slot. As expected, the capacity increases significantly when $\mathrm{TN}$ transmits a pulse train to represent bit 1 and broadcasting makes sense in terms of communications.

\section{Electrically Controllable Routing}

Semiconductor quantum dots (QD) are extensively used in FRET-based studies owing to their exceptional properties such as size-dependent tunable bandgap, high quantum yield and broad absorption spectrum as well as narrow emission spectrum. Another interesting property of QDs is Quantum Confined Stark Effect (QCSE) which defines the effect of external electric field on the optical properties of QDs [7].

The emission spectra of QDs are shifted in the red direction with the application of an electric field. The shift is named quantum confined stark shift and its relation with the electric field in terms of the shift in the energy spectrum is approximated as a sum of a linear and quadratic function

$$
\Delta E=\mu \xi+\frac{1}{2} \alpha \xi^{2}
$$

where $\Delta E$ is the shift in the energy spectrum; $\mu$ is the projection of excited-state dipole; $\alpha$ is the polarizability measure, and $\xi$ is the applied electric field [7].

A control mechanism over FRET efficiency is proposed in [8] by exploiting QCSE. In [8], FRET between CdSe QDs coated with CdS and Cy5 derivative dye molecules with the appliance of external electric field is studied and switch behavior of the mechanism over FRET is experimentally demonstrated obtaining a typical $10 \mathrm{~nm}$ wavelength shift. The idea is based on tuning the degree of the spectral overlap 


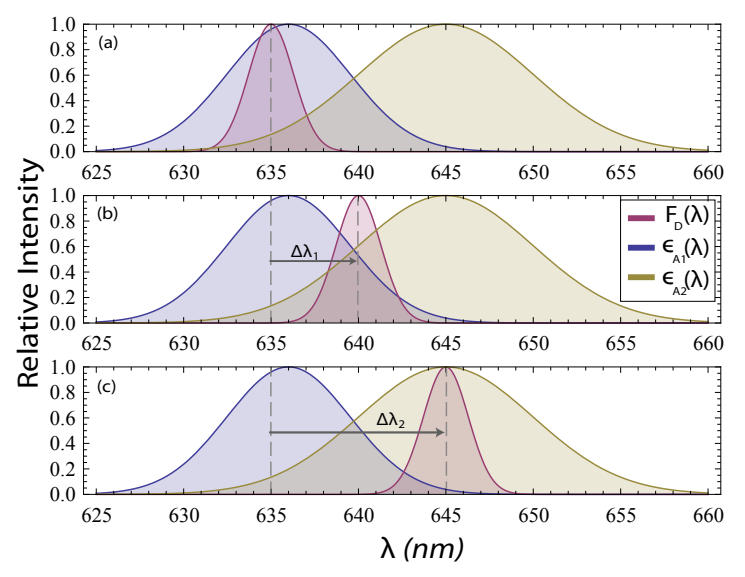

Fig. 5. Approximated emission spectrum of single CdSe and hypothetically modeled absorption spectra of the dye molecules at different spectral shifts (a) $\Delta \lambda=0$, (b) $\Delta \lambda=5 \mathrm{~nm}$, (c) $\Delta \lambda=10 \mathrm{~nm}$.

between donor $\mathrm{CdSe} / \mathrm{CdS}$ and acceptor $\mathrm{Cy} 5$ by changing the magnitude of the applied electric field. As the electric field increases, the emission spectrum of $\mathrm{CdSe} / \mathrm{CdS}$ is red-shifted, however, the absorption spectrum of the dye molecule is not affected by the electric field. Thus, the overlap either increases or decreases depending on the relative positions of the emission and absorption spectra of $\mathrm{CdSe} / \mathrm{CdS}$ and $\mathrm{Cy} 5$, respectively. As a result, efficiency is tuned with electric field.

In this study, we further develop the idea presented in [8] by approaching from the communication perspective and exploiting QCSE in order to design an electrically controllable router which routes the incoming information packets through one of the FRET channels making the selection according to the magnitude of the applied electric field. This type of router brings the advantage of routing the information flow at nanoscale by controlling it at macroscale.

In the model, the router nanomachine $(\mathrm{RtN})$ is considered as the first destination of the information coming from IS. Two RNs are considered to be located near RtN in the opposite sides with a distance $R$ to RtN as demonstrated in Fig. 4. Two dye molecules with different spectral properties are bound on RNs, and RtN is equipped with single QD of CdSe.

In order to investigate the operation of the router in terms of communication capacity, we approximate the emission and absorption spectra of CdSe and dye molecules, i.e., $F_{D}(\lambda)$, $\epsilon_{A 1}(\lambda)$ and $\epsilon_{A 2}(\lambda)$, by a single Gaussian function given as

$$
f(\lambda)=m \times \exp \left(-k\left(\lambda-\lambda_{c}\right)^{2}\right)
$$

where $m$ and $k$ are fitting parameters, $\lambda_{c}$ is the wavelength at which the emission or absorption intensity is maximum. $\lambda_{c}$ for single CdSe is approximated as $635 \mathrm{~nm}$ with zero electric field and it is shifted in the red direction with the appliance of an electric field, thus, $\lambda_{c, C d S e}$ is equal to $635+\Delta \lambda$, where $\Delta \lambda$ is the amount of the spectral shift in nanometers. $k$ gives a measure of the spectral broadening and it is set to $0.03 \mathrm{~nm}^{-2}$ for CdSe. $m$ is made equal to 1 for all the molecules. The absorption spectra of dye molecules are

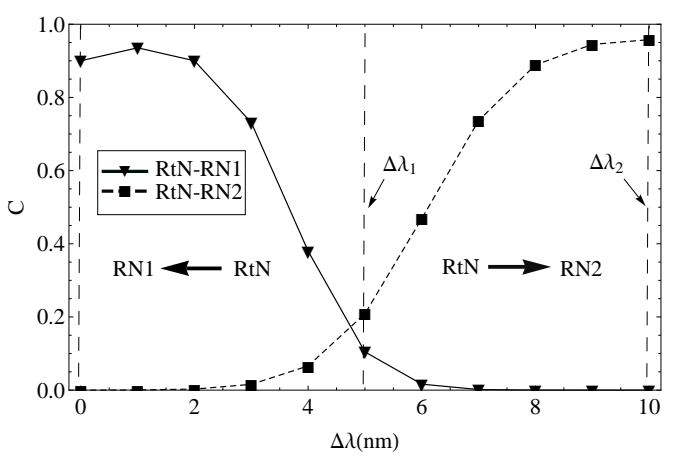

Fig. 6. Capacities of the channels TN-RN1 and TN-RN2 with varying $\Delta \lambda$. For low values of $\Delta \lambda$, the information flows only through RN1, however, for high values of $\Delta \lambda$, the information flow is through RN2.

designed hypothetically with $\lambda_{c, 1}=636 \mathrm{~nm}, \lambda_{c, 2}=645 \mathrm{~nm}$, $k_{1}=0.04 \mathrm{~nm}^{-2}$ and $k_{2}=0.02 \mathrm{~nm}^{-2}$.

The approximated spectra of the CdSe and dye molecules for different spectral shifts are demonstrated in Fig. 5. At zero electric field, the spectral shift is also zero and $A_{1}$ and CdSe have a significant spectral overlap, however, the overlap of $\mathrm{A}_{2}$ and CdSe is negligible. As the spectral shift increases with increasing electric field, the spectral overlap of $\mathrm{A}_{1}$ and CdSe first increases, and then decreases with increasing spectral shift; conversely, the spectral overlap of CdSe and $\mathrm{A}_{2}$ increases. The spectral overlap and Förster distance are directly related [5],

$$
R_{0}^{6} \propto \int_{0}^{\infty} F_{D}(\lambda) \epsilon_{A}(\lambda) \lambda^{4} \mathrm{~d} \lambda
$$

As a consequence of this relation, $p_{1}$ and spectral overlap are in positive correlation.

In the routing model, bit 1 is represented with four pulses with picosecond-durations, i.e., $\mathrm{N}=4$, and bit 0 is represented as silence during a time slot. The slot duration $t_{h}$ is equal to $4 T_{H}$ and $T_{H, \min }=\tau_{D, C d S e}+\tau_{A, \max }$ as in the case of broadcast communication. The distance of the RtN to both of the RNs is set to $R_{0, D-A 1}$ which is the Förster distance of CdSe- $\mathrm{A}_{1}$ pair when the electric field is zero. At these conditions, the dependence of the capacity on the spectral shift is demonstrated in Fig. 6. As is seen, RtN-RN1 channel capacity is near maximum at zero electric field and decreases to negligible values with increasing shift; conversely, RtN-RN2 channel capacity is negligible at zero electric field, however, it increases to its maximum as shift increases to $10 \mathrm{~nm}$. Therefore, around the zero electric field, the information is transmitted only to RN1; conversely, RtN transmits only to $\mathrm{RN} 2$, when the shift is around $10 \mathrm{~nm}$.

\section{A COMPlementary Network Analysis}

In this section, a comprehensive example of FRET-based communication network comprising broadcasting and routing concepts, as well as relaying concept defined in [4], is investigated. The network consists of one TN and four RNs which are connected through several relays (HN) and an electrically controlled RtN in an arrangement as seen in Fig. 7. 


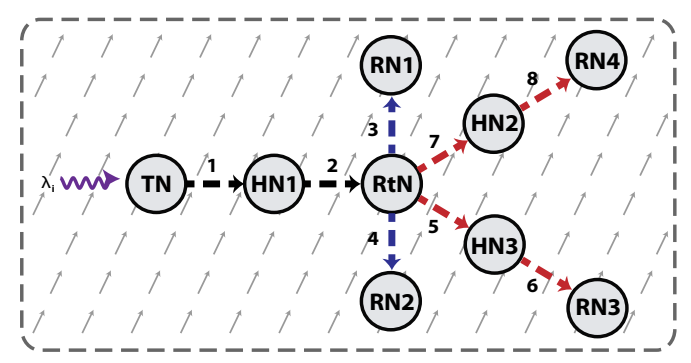

Fig. 7. A network scheme consisting of one TN, one RtN and several HNs and RNs with arrows indicating the possible routes of the information flow.

$A_{1}$ and $A_{2}$ molecules which are hypothetically designed in Section III are employed on HN2-HN3 and RN1-RN2 pairs, respectively. Except RtN, dye molecules are bound on the nanomachines. QD CdSe is bound on RtN. Förster distances between the molecules on the adjacent nodes except RtN-RN1 and RtN-RN2 pairs are assumed to be same and equal to $R_{0}$ at zero electric field. The internodal distance between any adjacent nodes are fixed to $0.8 R_{0}$. The spectral overlaps of the molecules on RtN-RN1 and RtN-RN2 pairs at zero electric field are negligibly small and increases with increasing electric field. FRET is allowed to occur through only the pathways indicated by arrows by assuming the length of other internodal pathways are sufficiently large, i.e., greater than $2 R_{0}$.

Relay nanomachines are excited by preceding excited molecule through FRET, and either fluorescence or transfer the excited state to the next molecule through FRET as described in [4]. The channels before and after HN constitute a series of independent FRET-channels; therefore, the probability of successful transmission of bit 1 for the relayed transmissions is given as $p_{1,12}=p_{1,1} \times p_{1,2}, p_{1,56}=p_{1,5} \times p_{1,6}$, $p_{1,78}=p_{1,7} \times p_{1,8}$, where $p_{1, i}$ represents $p_{1}$ for the $i^{t h}$ channel and $p_{1, i j}$ represents $p_{1}$ for the series of $i^{t h}$ and $j^{t h}$ channels.

At zero electric field, CdSe on RtN overlaps with the dye molecules on HN2 and HN2, while there is no spectral overlap between CdSe and the dye molecules on RN1 and RN2, thus, information is only broadcasted to HN2 and HN3. An electric field that is sufficiently large to shift the spectrum of CdSe around $10 \mathrm{~nm}$ results in a significant overlap of the spectra of CdSe and the dye molecules on RN1 and RN2, while reduces the overlap with the dye molecules on HN2 and HN3 into a negligible level. At this level of spectral shift, RtN broadcasts the information to RN1 and RN2 and the channels 5, 6, 7 and 8 do not carry any information. Therefore, switching between two network states is established.

Bit 1 and bit 0 are represented as $\mathrm{N}$ successive pulses and silence during a slot time $t_{H}$, respectively. Note that, $t_{H}=N \times T_{H}$. Determining the $T_{H}$, we have to consider the longest pathway that causes the longest delay in the network. $T_{H, \min }$ can be approximated as the sum of the lifetimes of the molecules employed on the longest path.

Assuming $t_{H} \geq N \times T_{H, \min }$, the capacity of the communication between TN and each RN is analyzed for different

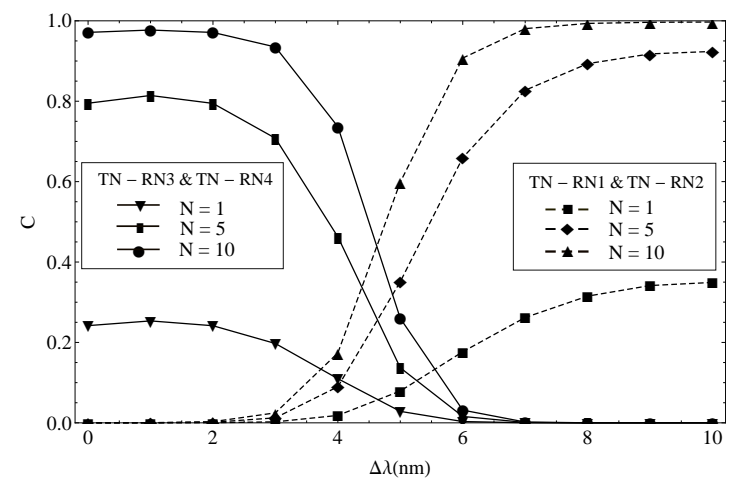

Fig. 8. Source-to-sink communication capacities of TN-RN1 and TN-RN2 as well as TN-RN3 and TN-RN4 pairs with varying $\Delta \lambda$ for different $N$.

numbers of pulses in the pulse train, varying the spectral shift between 0-10 nm and the results are shown in Fig. 8 . As is seen, it is possible to realize a communication with high reliability on a complex nanonetwork with the ability of electrically routing the information flow.

\section{CONClusion}

In this study, FRET-based nanoscale broadcast communication channel is investigated with the appliance of a pulse train waveform. Although the broadcast channel capacity is not adequate for reliable communication with the single pulse excitation, it is shown that, representing bit 1 with a laser pulse train significantly increases the broadcast channel capacity, and broadcasting makes sense from the communication point of view. Additionally, an electrical control mechanism over the route of the information flow in FRET-based nanonetworks is proposed exploiting QCSE. The mechanism brings the advantage of routing the information among several nanomachines with different operational capabilities. With the further advances in nanotechnology, we believe that FRET-based nanonetworking concepts some of which are proposed in this study will be extensively used in several nanoscale applications ranging from nuclear, biological and chemical defense to drug delivery and water quality control.

\section{REFERENCES}

[1] I. F. Akyildiz, F. Brunetti, C. Blazquez, "Nanonetworks: A new communication paradigm," Comput. Netw., vol. 52, no. 12, pp. 2260-2279, 2008.

[2] I. F. Akyildiz, M. J. Jornet, "Electromagnetic wireless nanosensor networks," Nano Commun. Netw., vol. 1, no. 1, pp. 3-19, 2010.

[3] B. Atakan, O. B. Akan, "Carbon nanotube-based nanoscale ad hoc networks," IEEE Commun. Mag., vol. 48, no. 6, pp. 129-135, 2010.

[4] M. Kuscu, O. B. Akan, "A physical channel model and analysis for nanoscale molecular communications with förster resonance energy transfer (FRET)," IEEE Trans. Nanotechnol., vol. 11, no. 1, pp. 200-207, 2012.

[5] J. R. Lakowicz, Principles of Fluorescence Spectroscopy, 3rd ed., Baltimore, MD: Springer-Verlag, 2006.

[6] E. A. Jares-Erijman, T. M. Jovin, "FRET imaging," Nature Biotechnol., vol. 21, pp. 1387-1395, 2003.

[7] S. A. Empedocles, M. G. Bawendi, "Quantum-confined stark effect in single CdSe nanocrystallite quantum dots," Science, vol. 278, pp. 21142117, 1997.

[8] K. Becker, J. M. Lupton, J. Müller, A. L. Rogach, D. V. Talapin, H. Weller, J. Feldmann, "Electrical control of Förster energy transfer," Nature Materials, no. 5, pp. 777-781, 2006. 